\title{
Perioperative Care of an Infant With an Open Safety Pin in the Hypopharynx
}

\author{
Arvid Yunga $^{\mathrm{a}}$, Haleh Saadat ${ }^{\mathrm{a}, \mathrm{b}, \mathrm{e}}$, Jonathan M. Grischkan ${ }^{\mathrm{c}}$, Joseph D. Tobias ${ }^{\mathrm{a}, \mathrm{b}, \mathrm{d}}$
}

\begin{abstract}
In the practice of pediatric anesthesia, foreign body (FB) aspiration represents a commonly encountered surgical emergency, which may lead to morbidity and mortality if not handled in a timely and effective fashion. We present a 12-month-old infant who was found to have a sharp FB lodged in the hypopharynx at the level of the glottis, leading to a unique clinical challenge, both from the standpoint of airway management and removal technique. We discuss the symptoms and radiographic manifestations of FB aspiration. Options for anesthetic management are presented and potential complications are reviewed.
\end{abstract}

Keywords: Foreign body; Aspiration; Anesthesia; Management; Considerations

\section{Introduction}

Foreign body (FB) aspiration refers to the inhalation of exogenous material into the larynx, trachea or bronchus. In the practice of pediatric anesthesia, FB aspiration represents a commonly encountered surgical emergency, which may lead to morbidity and mortality if not handled in a timely and effective fashion [1-5]. Undiagnosed or delayed treatment can result in pneumonia, atelectasis, lung abscess or fatal airway obstruction [6-8]. The annual incidence of death from FB aspiration has been estimated to be between 500 and 2,000 cases

Manuscript accepted for publication December 03, 2014

aDepartment of Anesthesiology and Pain Medicine, Nationwide Children's Hospital, Columbus, OH, USA

${ }^{b}$ Department of Anesthesiology and Pain Medicine, The Ohio State University College of Medicine, Columbus, OH, USA

'Department of Otolaryngology-Head and Neck Surgery, Nationwide Children's Hospital and The Ohio State University, Columbus, OH, USA

dDepartment of Pediatrics, The Ohio State University College of Medicine, Columbus, OH, USA

${ }^{\mathrm{e} C}$ Corresponding Author: Haleh Saadat, Department of Anesthesiology and Pain Medicine, Nationwide Children's Hospital, 700 Children's Drive, Columbus, OH 43205, USA. Email: Haleh.Saadat@nationwidechildrens.org

doi: http://dx.doi.org/10.14740/jmc2017w per year in the United States. Therefore, the timely diagnosis and management are crucial in ensuring a successful outcome. We report the perioperative care of a 12-month-old infant who presented with an open safety pin in the hypopharynx near the glottic opening. Options for anesthetic care including mode of ventilation (controlled versus spontaneous) are discussed.

\section{Case Report}

Institutional Review Board approval is not required for single patient case report at Nationwide Children's Hospital (Columbus, $\mathrm{OH}$ ). A previously healthy, 12-month-old, $11.75 \mathrm{~kg}$ girl presented to the Emergency Department (ED) with suspicion of an FB aspiration. The mother suspected that she had seen her swallowing something, but was unsure of the nature of the material. The child immediately started to be irritable with emesis and drooling. There was no evidence of respiratory compromise or stridor. The mother immediately called Emergency Medical Services (EMS), and the child was brought to the ED. On examination, there were no signs of choking, stridor or difficulty breathing. The patient was managing her own secretions well. Her past medical history was negative with no prior surgery or health problems. The family history was positive only for factor $\mathrm{V}$ Leiden in the mother. FB radiographs demonstrated an open safety pin, which appeared to be embedded in the posterior pharyngeal soft tissues at the level of the adenoids (Fig. 1). Based on this, recommendation was made to proceed to the operating room for direct laryngoscopy, bronchoscopy and hypopharyngeal FB removal. The patient was transported to the operating room and routine American Society of Anesthesiologists' monitors were applied. Anesthesia was induced with incremental concentrations of sevoflurane (1-8\%) in $100 \%$ oxygen. Following anesthetic induction, a peripheral intravenous cannula was placed. A total of $30 \mathrm{mg}$ of propofol was administered in $10 \mathrm{mg}$ increments while maintaining spontaneous ventilation prior to airway instrumentation. No opioids were administered, but acetaminophen (10 $\mathrm{mg} / \mathrm{kg}$ ) was administered intravenously for postoperative analgesia. Once an adequate depth of anesthesia was achieved, the table was rotated $90^{\circ}$, a shoulder roll was placed and the pediatric otolaryngologist used an operating/ventilating laryngoscope to expose the larynx. Spontaneous ventilation was maintained throughout the procedure. Oxygen and sevoflu- 


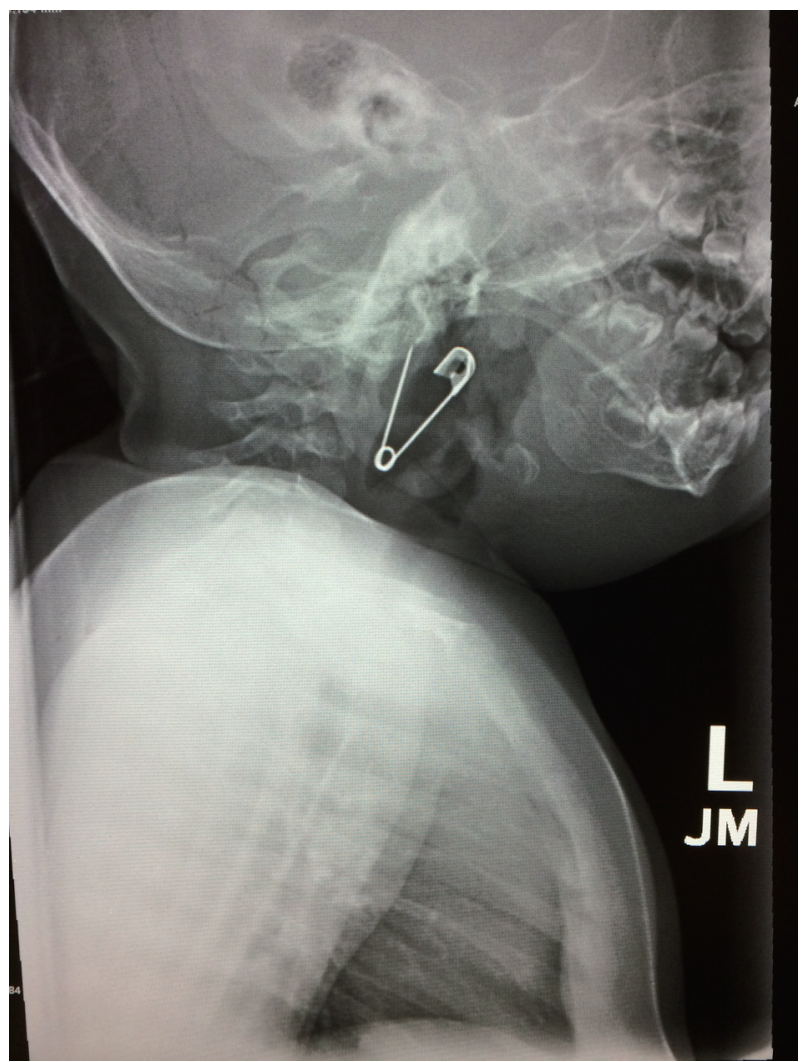

Figure 1. Preoperative radiographs demonstrating an open safety pin in the posterior pharyngeal soft tissues at the level of the adenoids.

rane were insufflated into the oropharynx via the laryngoscope side-port to maintain an adequate depth of anesthesia. The zero degree Hopkins rod telescope was used for visualization, and the safety pin was identified in the hypopharynx (Fig. 2). The open pointed end was embedded in the right pharyngeal wall mucosa along the posterior pharyngeal at the junction of the lateral pharyngeal wall. The pointed end of the pin was grasped with alligator forceps, pushed inferiorly and removed from the pharyngeal mucosa. The other end was grasped and FB was pulled out with the pointed end facing down. The zero degree telescope was then used to examine the larynx, subglottic area, trachea and the main stem bronchi which were all normal in appearance. Spontaneous ventilation was maintained throughout the procedure, and the patient was allowed to awaken from general anesthesia in the operating room. Additional intraoperative medications included dexamethasone $(0.3 \mathrm{mg} / \mathrm{kg})$ and ondansetron $(0.1 \mathrm{mg} / \mathrm{kg})$. The patient was transported to the post-anesthesia care unit in stable condition. She was admitted to the inpatient ward for monitoring of her postoperative respiratory status with continuous pulse oximetry. The remainder of her postoperative course was unremarkable, and she was discharged home later the same day without incident.

\section{Discussion}

In the United States, asphyxia from choking and suffocation is

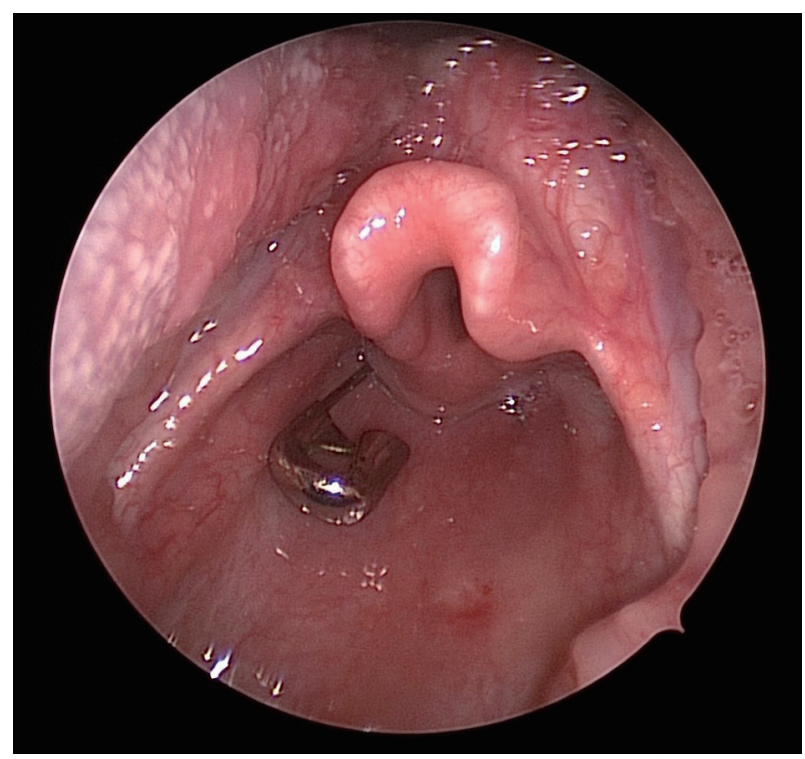

Figure 2. Intraoperative photograph with the safety pin in the hypopharynx.

the third most common cause of home and community death. The majority of FB aspirations occur in young children under 3 years of age $[9,10]$. Food matter (hot dogs, popcorn, hard candy, peanut butter, etc.) is the substance that is most commonly aspirated; however, many other small household items and toys such as latex balloons, coins, marbles, small balls, crayons, rings and ornaments may be involved $[4,5]$. Occasionally, as was noted in our patient, sharp objects such as pins, nails metallic bristles may be aspirated. An FB that passes the larynx can lodge anywhere in the tracheobronchial tree, but most commonly pass into the right mainstem bronchus as the angle between the trachea and the right main bronchus is less acute.

The presenting symptoms of FB aspiration or ingestion are often nonspecific, requiring a high degree of suspicion to make an accurate diagnosis. In some scenarios, the parents will report feeding or breathing problems without a specific history suggesting FB ingestion. The symptoms may range from none to severe airway obstruction. Alternatively, if untreated, clinical signs of infection including pneumonia or lung abscess can develop months after the aspiration event. Although a history of a witnessed choking or gagging episode is crucial, such a history is not always available. In the absence of the classic triad of choking, cough and wheezing, the diagnosis may be delayed for weeks to months after going through multiple rounds of medical therapy, only to be discovered during the evaluation for prolonged respiratory complications. Other presentations include neck and/or throat pain, fever, dyspnea, hoarseness, perioral cyanosis and retractions. At times, the radiologist may be the first one to raise the possibility of an FB.

An FB near the glottic inlet, as described in our patient, may result in acute airway distress, hoarseness and stridor. These may result in asphyxia and cardiac arrest at the time of aspiration or emergent presentation to the ED. Severe airway 
obstruction may require emergent transportation to the operating room for direct laryngoscopy with bronchoscopy for removal of the FB. Although our patient's presentation was acute with drooling and irritability likely occurring immediately after aspirating the FB, there were no signs of immediate airway compromise and the diagnosis was made based on clinical findings with history and further confirmed by the initial radiography.

Traditionally, definitive diagnosis and treatment involve rigid bronchoscopy after the induction of anesthesia. The care of such patients begins prior to their arrival with the development of an acute airway algorithm to deal with a pediatric patient with a compromised airway. Such practices were commonly in place in the era of acute epiglottis where such presentations were seen more frequently. However, it has been our experience with the rapid decline in epiglottitis following widespread immunization for Haemophilus influenza, the familiarity with such pathways has declined [11]. Therefore, we would suggest that these algorithms be in place to allow the immediate identification of such patients and the notification of the required services including anesthesiology and otolaryngology. Additional guidelines may include reminders of the need to keep the patient calm, avoidance of painful procedures including peripheral intravenous cannulation and a rapid pathway for transport to the operating room. The decision must also be made regarding the stability of the patient's airway and the need for radiographic imaging. Transportation to the operating room should be performed with the necessary personnel and equipment at the bedside. In our institution, this includes the anesthesia provider and the surgeon in addition to other operating room personnel.

As was commonplace in the era of acute epiglottitis, the induction of anesthesia includes sevoflurane in 100\% oxygen with an intravenous cannula generally placed after anesthetic induction $[12,13]$. This is performed regardless of the nil per os status. While there is limited evidence-based medicine to guide practice, options available to potentially decrease the risk of aspiration of gastric contest include the application of cricoid pressure during inhalation induction (after the loss of consciousness) or placement of an orogastric tube with gastric suctioning after induction, but before the bronchoscope is inserted. We would recommend that the surgeon be present at the bedside during inhalational induction, ready for a potential surgical airway intervention if needed. Inhalational induction and maintenance of spontaneous ventilation is generally considered the technique of choice as it prevents further migration of FB into the airway which may theoretically occur with positive pressure ventilation $[14,15]$. However, the validity of this assumption has been questioned in a retrospective review of airway management for $94 \mathrm{FB}$ aspirations in infants and children [16]. Forty-seven patients were managed by controlled ventilation, 18 by assisted ventilation and 26 by spontaneous ventilation. The management of three patients could not be determined because of insufficient information on the chart and were excluded from analysis. The charts were reviewed for various adverse events including hypoxemia, hypercarbia, bradycardia, hypotension and the need for oxygen supplementation in the post-anesthesia care unit. The authors concluded that none of the adverse events were associated with a particu- lar ventilation technique.

Our general practice remains the induction of anesthesia using sevoflurane in $100 \%$ oxygen with the maintenance of spontaneous ventilation. With a cooperative patient, pre-oxygenation with $100 \%$ oxygen should be attempted. Given that it has limited utility as far as speeding induction when using sevoflurane, we generally do not use nitrous oxide in such cases. Nitrous oxide also dilutes the inspired oxygen concentration thereby providing less time if effective ventilation is lost. If available, helium may be used to decrease gas density, increase flow and speed anesthetic induction in patients with a compromised airway and decreased minute ventilation [17, 18]. In the majority of cases, anesthesia is maintained with the insufflation of sevoflurane via the operating laryngoscope, although others have described the use of various intravenous anesthetic agents including remifentanil, propofol and dexmedetomidine $[15,19,20]$. Each of these techniques has their limitations. The insufflation of an inhalational agent can cause significant environmental pollution and healthcare worker exposure while intravenous techniques may lead to hypercarbia or apnea. Although less commonplace than with halothane, significant cardiovascular compromise may occur during the administration of a high concentration of sevoflurane (6-8\%) for a prolonged period of time [21].

Depth of anesthesia should be ensured before instrumentation of the airway (vital signs, respiratory pattern, pupil size, muscle tone). The application of a local anesthetic solution on the larynx and trachea can decrease the potential for coughing upon introduction of the bronchoscope. In our practice, this includes the use of $1-2 \%$ lidocaine ( $4-5 \mathrm{mg} / \mathrm{kg}$ ) applied using a mucosal atomizing device. This is performed under direct vision by the surgeon prior to placement of the bronchoscope. During rigid bronchoscopy, an inadequate depth of anesthesia manifested as an increase in heart rate, respiratory rate or movement can be treated by incremental doses of propofol (1 $\mathrm{mg} / \mathrm{kg}$ ) while maintaining spontaneous ventilation.

Postoperative care depends on the operative course of the patient. Ideally, FB removal would result in little to no trauma. However, soft tissue and tracheal trauma can occur. Stridor and wheezing are two common adverse effects from instrumentation of the airway resulting in soft tissue edema. This is particularly true in cases necessitating complex or prolonged airway manipulation. For this reason, the patient's respiratory status should be closely monitored postoperatively. It is routine in our practice to administer intravenous corticosteroids (dexamethasone) during the procedure to minimize postoperative stridor resulting from subglottic edema. Racemic epinephrine may also be beneficial postoperatively. FB fragmentation and infection can predispose the patient to postoperative wheezing. Nebulized albuterol and chest physiotherapy may improve symptoms by aiding in clearance of foreign material. Antibiotics are indicated if infection is suspected or purulent drainage seen during airway endoscopy.

In summary, the tendency of children to put non-food objects in their mouths is dangerous and potentially life-threatening. FB aspiration remains a commonly encountered surgical emergency in the pediatric population. A suggestive history, physical exam and radiologic evidence are important in aiding the diagnosis of FB aspiration. Definitive diagnosis and treat- 
ment involve rigid bronchoscopy under general anesthesia. Anesthetic management should be undertaken by experienced physicians, and all equipment should be checked before starting. Ensuring an adequate depth of anesthesia before airway instrumentation is also important in reducing further migration of FB and preventing airway compromise with coughing or laryngospasm. Inhalational induction with sevoflurane in $100 \%$ oxygen remains the common anesthetic technique; however, the use of intravenous anesthetic agents has also been described.

\section{References}

1. Kadmon G, Stern Y, Bron-Harlev E, Nahum E, Battat E, Schonfeld T. Computerized scoring system for the diagnosis of foreign body aspiration in children. Ann Otol Rhinol Laryngol. 2008;117(11):839-843.

2. Eren S, Balci AE, Dikici B, Doblan M, Eren MN. Foreign body aspiration in children: experience of 1160 cases. Ann Trop Paediatr. 2003;23(1):31-37.

3. Zhijun C, Fugao Z, Niankai Z, Jingjing C. Therapeutic experience from 1428 patients with pediatric tracheobronchial foreign body. J Pediatr Surg. 2008;43(4):718721.

4. Cohen SR, Herbert WI, Lewis GB, Jr., Geller KA. Foreign bodies in the airway. Five-year retrospective study with special reference to management. Ann Otol Rhinol Laryngol. 1980;89(5 Pt 1):437-442.

5. Mantel K, Butenandt I. Tracheobronchial foreign body aspiration in childhood. A report on 224 cases. Eur J Pediatr. 1986;145(3):211-216.

6. Inglis AF, Jr., Wagner DV. Lower complication rates associated with bronchial foreign bodies over the last 20 years. Ann Otol Rhinol Laryngol. 1992;101(1):61-66.

7. Zaytoun GM, Rouadi PW, Baki DH. Endoscopic management of foreign bodies in the tracheobronchial tree: predictive factors for complications. Otolaryngol Head Neck Surg. 2000;123(3):311-316.

8. Farrel PT. Rigid bronchoscopy for foreign body removal: anesthesia and ventilation. Pediatr Anesth. 2004;14:8489.

9. Chiu CY, Wong KS, Lai SH, Hsia SH, Wu CT. Factors predicting early diagnosis of foreign body aspiration in children. Pediatr Emerg Care. 2005;21(3):161-164.

10. Tan HK, Brown K, McGill T, Kenna MA, Lund DP, Healy GB. Airway foreign bodies (FB): a 10-year review. Int J Pediatr Otorhinolaryngol. 2000;56(2):91-99.

11. Gorelick MH, Baker MD. Epiglottitis in children, 1979 through 1992. Effects of Haemophilus influenzae type $b$ immunization. Arch Pediatr Adolesc Med. 1994;148(1):47-50.

12. Bottenfield GW, Arcinue EL, Sarnaik A, Jewell MR. Diagnosis and management of acute epiglottitis -- report of 90 consecutive cases. Laryngoscope. 1980;90(5 Pt 1):822-825.

13. Hannallah R, Rosales JK. Acute epiglottitis: current management and review. Can Anaesth Soc J. 1978;25(2):8491.

14. Rovin JD, Rodgers BM. Pediatric foreign body aspiration. Pediatr Rev. 2000;21(3):86-90.

15. Liao R, Li JY, Liu GY. Comparison of sevoflurane volatile induction/maintenance anaesthesia and propofol-remifentanil total intravenous anaesthesia for rigid bronchoscopy under spontaneous breathing for tracheal/ bronchial foreign body removal in children. Eur J Anaesthesiol. 2010;27(11):930-934.

16. Litman RS, Ponnuri J, Trogan I. Anesthesia for tracheal or bronchial foreign body removal in children: an analysis of ninety-four cases. Anesth Analg. 2000;91(6):13891391, TOC.

17. Tobias JD. Helium insufflation with sevoflurane general anesthesia and spontaneous ventilation during airway surgery. Can J Anaesth. 2009;56(3):243-246.

18. Tobias JD. Helium: Applications in the practice of anesthesia and critical care. Am J Anesthesiol. 1997;24:194200.

19. Ansermino JM, Brooks P, Rosen D, Vandebeek CA, Reichert C. Spontaneous ventilation with remifentanil in children. Paediatr Anaesth. 2005;15(2):115-121.

20. Shukry M, Kennedy K. Dexmedetomidine as a total intravenous anesthetic in infants. Paediatr Anaesth. 2007;17(6):581-583.

21. Ramamoorthy C, Haberkern CM, Bhananker SM, Domino KB, Posner KL, Campos JS, Morray JP. Anesthesiarelated cardiac arrest in children with heart disease: data from the Pediatric Perioperative Cardiac Arrest (POCA) registry. Anesth Analg. 2010;110(5):1376-1382. 